- Clinical Research •

\title{
Impact of adjuvant chemotherapy duration on 3-year disease-free survival of colorectal carcinoma patients after radical resection
}

\author{
Miao-Zhen Qiu, , 1,2 Kai-Yuan Teng, 1,2 Dan-Yun Ruan, ${ }^{1,2}$ Yu-Hong Li , , ${ }^{1,2}$ Xiao-Qin Chen , 1,2 \\ Zhi-Zhong Pan, ${ }^{1,3}$ You-Jian He, ${ }^{1,2}$ De-Sen Wan ${ }^{1,3}$ and Rui-Hua $X_{u^{1,2}}$
}

\author{
1. State Key Laboratory of \\ Oncology in South China, \\ Guangzhou, Guangdong, 510060 , \\ P. R. China \\ 2. Department of Medical \\ Oncology, \\ Sun Yat-sen University Cancer \\ Center, \\ Guangzhou, Guangdong, 510060, \\ P. R. China \\ 3. Department of Abdominal \\ Surgery, \\ Sun Yat-sen University Cancer \\ Center, \\ Guangzhou, Guangdong, 510060 , \\ P. R. China
}

Correspondence to: Rui-Hua Xu
Tel.: 86.20 .87343468
Fax : 86.20.87343468
Email : xurh@mail.sysu.edu.cn

This paper was translated into English from its original publication in Chinese.

Translated by: Miao-Zhen Qiu

and Wei Liu

The original Chinese version of this paper is published in:Ai Zheng (Chinese Journal of Cancer) 28(7);http://www.cjcsysu.cn/cn/ article.asp?id=15751 )

Submitted : 2008-12-09

Revised : 2009-03-10

\begin{abstract}
[Abstract] Background and Objective: Adjuvant chemotherapy has become a standard postoperative treatment for stage III and high risk stage II colorectal carcinoma patients. However, only a few patients can finish 6-month adjuvant chemotherapy. This study was to find out whether the duration of adjuvant chemotherapy would affect the 3-year disease-free survival. Methods: Clinical data of 276 colorectal carcinoma patients, receiving at least two cycles of adjuvant chemotherapy including xeloda, 5-fluorouracil/calcium folinate (5-FU/ CF) or Tegafur with or without oxaliplatin after radical operation in Sun Yat-sen University Cancer Center from April, 2003 to December, 2007, were analyzed for the impact of adjuvant chemotherapy duration on the 3-year disease-free survival. Results: Of the 276 patients, 216 received chemotherapy including oxaliplatin, 60 received xeloda, 5-FU/CF or tegafur as adjuvant chemotherapy. Of the 216 patients, only 49 finished the 6-month adjuvant chemotherapy. Both univariate and multivariate analyses showed that chemotherapy duration ( $P=$ $0.032)$, sex $(P=0.001)$, $N$ stage $(P=0.002)$, and pathologic differentiation ( $P=$ 0.043 ) were independent prognosis factors for 3-year disease-free survival. Conclusion: Duration of adjuvant chemotherapy is an independent prognosis factor for 3-year disease-free survival of colorectal carcinoma patients.
\end{abstract}

Key words: colorectal neoplasm, adjuvant chemotherapy, duration, disease-free survival

Colorectal cancer is a common cancer with an increasing incidence. In 2002, about 1023000 cases of colorectal cancer were reported globally. Cao et al. ${ }^{1}$ reported that during 2000-2002, the incidence and mortality of colorectal cancer in Guangzhou ranked fourth and sixth for men, fifth and seventh for women, respectively. Surgical resection is the main treatment for colorectal cancer. However, many stage II and III patients develop recurrence and metastasis after operation. The 2008 NCCN guidance recommended 6 months of postoperative adjuvant chemotherapy for stage III patients or stage II patients at high risk, ${ }^{2,3} \mathrm{We}$ found that only some of these patients finished 6-month adjuvant chemotherapy. This study was to explore factors that affect patients compliance, the impact of duration of adjuvant chemotherapy on disease-free survival, and the efficacy of 6-month chemotherapy of 
5-fluorouracil (5-FU)/calcium folinate (CF) or xeloda on the patients discontinued treatment of oxaliplatin within 6 months.

\section{Materials and Methods}

Patients. Clinical data of 276 patients with resectable colorectal carcinoma, who were admitted to Sun Yat-sen University Cancer Center from July 2003 to December 2007, were analyzed. All the patients met the following criteria: over 18 years old, had pathologically confirmed colorectal carcinoma, received radical resection, at stage III (T1-4, N1-3) or high-risk stage II [high-risk factors included poor histological differentiation, T4 tumors, bowel obstruction or perforation, indeterminate or positive margins, peritumoral lymphovascular involvement, and inadequately $(<12)$ sampled nodes], ${ }^{3,4}$ received at least 2 cycles of adjuvant chemotherapy including 5-FU/CF, or xeloda, or tegafur with or without oxaliplatin, with detailed follow-up information. Patients who received preoperative neoadjuvant radiochemotherapy were excluded. Totally 276 patients were suitable for the final analysis: $151(54.7 \%)$ were at stage III, $125(45.3 \%)$ at high risk stage II. The clinical data of the patients are listed in Table 1.

Methods. Of the 276 colorecal carcinoma patients, 216 received adjuvant chemotherapy including oxaliplatin. Among them, 104 received mFOLFOX6 regimen (ranging from 2 to 12 cycles, with a median of 6 cycles), 93 received XELOX regimen (ranging from 2 to 10 cycles, with a median of 6 cycles), 7 received mFOLFOX6 regimen at first and shifted to XELOX regimen due to the falling off of the PICC tube or infection, 12 primarily received XELOX regimen and changed to mFOLFOX6 regimen because of the hand-foot syndrome or a poor economic situation.

mFOLFOX6 regimen was given as follows: oxaliplatin $\left(85-100 \mathrm{mg} / \mathrm{m}^{2}\right)$ at day 1 , bolus of 5 -FU $\left(400 \mathrm{mg} / \mathrm{m}^{2}\right)$ at day 1 , continuous intravenous of 5-FU $\left(2400-3000 \mathrm{mg} / \mathrm{m}^{2}\right)$ for 46 $\mathrm{h}$, and CF $\left(400 \mathrm{mg} / \mathrm{m}^{2}\right)$ for $2 \mathrm{~h}$ at day 1 , repeated every 14 to 18 days.

XELOX regimen was given as follows: oxaliplatin $\left(100-130 \mathrm{mg} / \mathrm{m}^{2}\right)$ at day 1 , and xeloda $\left(1700-2000 \mathrm{mg} / \mathrm{m}^{2}\right)$ at day $1-14$, repeated every 21-25 days.

The other 60 patients received adjuvant chemotherapy without oxaliplatin. Among them, 39 were at high-risk stage IIa, 14 at stage IIb, 6 at stage IIIb and 1 at stage IIIc; 35 were treated with xeloda (ranging from 3 to 8 cycles, with a median of 6 cycles), 9 were treated with 5-FU/CF (ranging from 3 to 10 cycles, with a median of 5 cycles), and 16 were treated with tegafur (ranging from 2 to 7 cycles, with a median of 5 cycles). The dose of xeloda was $1700-2000 \mathrm{mg} / \mathrm{m}^{2}, \mathrm{~d} 1-14$, repeated every $21-28$ days. 5-FU/CF regimen was given as follows: bolus of 5 -FU $\left(400 \mathrm{mg} / \mathrm{m}^{2}\right)$ at day 1 , continuous intravenous of 5-FU (2400-3000 $\left.\mathrm{mg} / \mathrm{m}^{2}\right)$ for $46 \mathrm{~h}, \mathrm{CF}\left(400 \mathrm{mg} / \mathrm{m}^{2}\right)$ at day 1 , repeated every 14-18 days. The dose of Tegafur was $200 \mathrm{mg}$, three times every day, four weeks on and 2 weeks off, repeated every 42-48 days.

Evaluation criteria for adverse events. Adverse events were assessed according to the NCI-CTCAE (National Cancer Institute-Common Terminology Criteria for Adverse Events) 3.0.5 Neurotoxicity of oxaliplatin was evaluated based on clinical records and patients description during follow-up.

Statistical analysis. All statistical analyses were done with SPSS16.0 software. All P values were 2-tailed, and the level of significance was set at 0.05 . Disease-free survival and overall survival were analyzed by Kaplan-Meier method. Differences were estimated with the log-rank test. Prognostic factors were examined by univariate and multivariate analyses using Cox proportional hazards regression model.

\section{Results}

Till October 2008, the median follow-up duration was 36.3 months (ranging from 2 to 74.5 months). Fifteen patients died of disease progression; 64 suffered from local recurrence or distant metastasis, among them $11(17.2 \%)$ had recurrence and $53(82.8 \%)$ had distant metastasis. In detail, $21(39.6 \%)$ patients had 
liver metastasis, 15 (28.3\%) had lung metastasis, $15(28.3 \%)$ had pelvic dissemination, 10 $(18.9 \%)$ had retroperitoneal lymph nodes or abdominal organ metastasis except liver metastasis, $4(7.5 \%)$ had inguinal or supraclavicular lymph node metastasis; 10patients had multiple metastases. The 3-year disease-free survival rate of the 276 patients was
$71.2 \%$ (Fig. 1) and the 5-year overall survival rate was $86.3 \%$ (Fig. 2).

Among the 104 patients who took mFOLFOX6 regimen, only $20(19.2 \%)$ finished 12 cycles of chemotherapy; $8(7.7 \%)$ received 5 -FU/CF or xeloda as maintenance till the end of 6-month chemotherapy after 6-11 cycles of mFOLFOX6 due to grade III neuropathy (4

Table 1 Clinical data and 3-year disease-free survival of the colorectal carcinoma patients

\begin{tabular}{|c|c|c|c|c|}
\hline Item & $\begin{array}{c}\text { Total } \\
{[\operatorname{cases}(\%)]^{\mathrm{a}}}\end{array}$ & $\begin{array}{l}\text { Chemotherapy including } \\
\text { oxaliplatin }[\text { cases }(\%)]^{\mathrm{b}}\end{array}$ & $\begin{array}{c}\text { 3-year DFS } \\
(\%)^{c}\end{array}$ & $P$ value \\
\hline \multicolumn{5}{|l|}{ Age } \\
\hline$<40$ & $45(16.3)$ & $37(17.2)$ & 51.0 & \\
\hline $40-49$ & $58(21.0)$ & $48(22.2)$ & 66.0 & \\
\hline $50-59$ & $91(33.0)$ & $74(34.3)$ & 70.2 & 0.043 \\
\hline $60-69$ & $55(19.9)$ & $42(19.4)$ & 89.3 & \\
\hline$\geqslant 70$ & $27(9.8)$ & $15(6.9)$ & 83.3 & \\
\hline \multicolumn{5}{|l|}{ Sex } \\
\hline Female & $110(39.9)$ & $82(38.0)$ & 81.6 & \\
\hline Male & $166(60.1)$ & $134(62.0)$ & 64.9 & 0.023 \\
\hline \multicolumn{5}{|l|}{ Site } \\
\hline Ileocecum & $13(4.7)$ & $7(3.2)$ & 100.0 & \\
\hline Ascending colon & $52(18.8)$ & $39(18.1)$ & 70.4 & \\
\hline Transverse colon & $25(9.1)$ & $18(8.3)$ & 69.4 & \\
\hline Descending colon & $29(10.5)$ & $19(8.8)$ & 81.4 & \\
\hline Sigmoid colon & $63(22.8)$ & $46(21.3)$ & 75.7 & \\
\hline Rectal colon & $93(33.7)$ & $86(39.8)$ & 64.4 & \\
\hline HNPCC & $1(0.4)$ & $1(0.5)$ & 100.0 & 0.453 \\
\hline \multicolumn{5}{|l|}{ Tumor size } \\
\hline$\leqslant 5 \mathrm{~cm}$ & $150(65.8)$ & $120(67.8)$ & 77.4 & \\
\hline$>5 \mathrm{~cm}$ & $78(34.2)$ & $57(32.2)$ & 75.4 & 0.938 \\
\hline \multicolumn{5}{|l|}{ TNM stage } \\
\hline II & $125(45.3)$ & $72(33.4)$ & 86.0 & \\
\hline III & $151(54.7)$ & $144(66.6)$ & 63.5 & 0.002 \\
\hline \multicolumn{5}{|l|}{ CEA expression } \\
\hline Negative & $12(5.8)$ & $11(6.9)$ & 55.6 & \\
\hline+ & $137(66.2)$ & $104(65.0)$ & 78.7 & \\
\hline++ & $26(12.6)$ & $21(13.1)$ & 78.3 & \\
\hline+++ & $32(15.5)$ & $24(15.0)$ & 70.3 & 0.593 \\
\hline \multicolumn{5}{|l|}{ Preoperative serum CEA level } \\
\hline Normal & $152(61.3)$ & $116(59.8)$ & 74.8 & \\
\hline Elevated & $96(38.7)$ & $78(40.2)$ & 74.6 & 0.893 \\
\hline \multicolumn{5}{|l|}{ Pathologic subtype } \\
\hline Poorly differentiated adenocarcinoma & $52(19.3)$ & $41(19.3)$ & 45.8 & \\
\hline Moderately differentiated adenocarcinoma & $190(70.6)$ & $149(70.3)$ & 79.6 & \\
\hline Well differentiated adenocarcinoma & $14(5.2)$ & $10(4.7)$ & 87.5 & \\
\hline Signet ring cell & $7(2.6)$ & $6(2.8)$ & 62.5 & \\
\hline Mucinous adenocarcinoma & $6(2.3)$ & $6(2.8)$ & 80.0 & 0.001 \\
\hline \multicolumn{5}{|l|}{ Duration of chemotherapy without maintenance } \\
\hline$\leqslant 2$ months & & $30(13.9)$ & 57.9 & \\
\hline $2-4$ months & & $81(37.5)$ & 65.5 & 0.058 \\
\hline $4-6$ months & & $105(48.6)$ & 78.6 & \\
\hline \multicolumn{5}{|l|}{ Duration of chemotherapy with maintenance } \\
\hline$\leqslant 2$ months & & $27(12.5)$ & 51.5 & 0.010 \\
\hline $2-4$ months & & $75(34.7)$ & 64.0 & \\
\hline $4-6$ months & & $114(52.8)$ & 79.4 & \\
\hline
\end{tabular}

DFS, disease-free survival; HNPCC, hereditary nonpolypsis colorectal carcinoma. anly 228, 207, 248 and 269 patients were included for


patients were included for analyses on tumor size, CEA expression, preoperative serum CEA level and pathologic subtype, respectively. ${ }^{\circ}$ Only the patients who received chemotherapy including oxaliplatin were included for analysis on 3-year disease-free survival. 
cases), grade IV neutropenia (1 case), and unknown reasons (3 cases).

Among the 93 patients who received XELOX regimen as adjuvant chemotherapy, $15(16.1 \%)$ finished 6-month chemotherapy; 5 took xeloda as maintenance till the end of 6-month chemotherapy after 2-7 cycles of XELOX due to allergy to oxaliplatin (2 cases), grade III neuropathy (2 cases), and unknown reason (1 case).

Among the 19 patients who used mFOLFOX plus XELOX regimen, only one finished 3 cycles of mFOLFOX6 and 3 cycles of XELOX, and took xeloda as maintenance due to grade III neuropathy.

Only 49 patients finished 6 months of adjuvant chemotherapy (including those who took 5-FU/CF or xeloda as maintenance till the end of 6-month chemotherapy). Among them only 35 finished 6 months of oxaliplatin-including regimens. The median adjuvant chemotherapy duration for the 167 patients who did not finish 6-month chemotherapy was 4 months. The reasons of chemotherapy discontinuation were as follows: the physicians decision (102 cases, 61.1\%), patients refusal (51 cases, 30.5\%), neuropathy ( 4 cases, 2.4\%), disease progression (4 cases, $2.4 \%$ ), hand-foot syndromes ( 2 cases, $1.2 \%$ ), grade IV neutropenia ( 1 case, $0.6 \%$ ), grade III diarrhea (1 case, $0.6 \%)$, allergy response to oxaliplatin ( 2 cases, $1.2 \%$ ).

The 216 patients who received oxaliplatin-including regimens were divided into 3 groups according to the duration of adjuvant chemotherapy (excluding the duration of xeloda or 5-FU/CF maintenance): $\leq 2$ months, 2-4 months (including 4 months), 4-6 months (including 6 months). The 3-year disease-free survival rates of these three groups were $57.9 \%$, $65.5 \%$ and $78.6 \%$, respectively $(\mathrm{P}=0.058)$ (Fig. 3). Including the duration of xeloda and 5-FU/CF maintenance when dividing the patients into three groups as above, the 3-year disease-free survival rates were $51.5 \%, 64.0 \%$ and $79.4 \%$, respectively $(\mathrm{P}=0.01)$ (Fig. 4).

Univariate analysis showed that age $(\mathrm{P}=$ 0.043), gender $(P=0.023)$, PS score $(P<0.001)$,

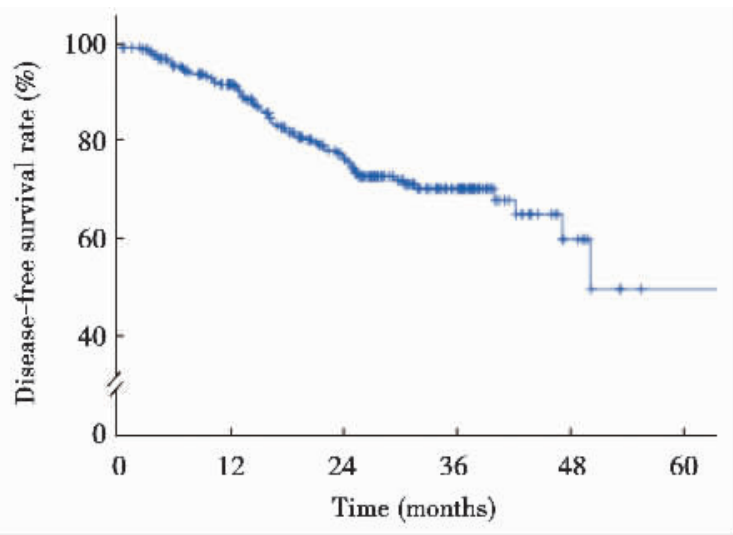

Figure 1 Disease-free survival curve of the 276 colorectal carcinoma patients

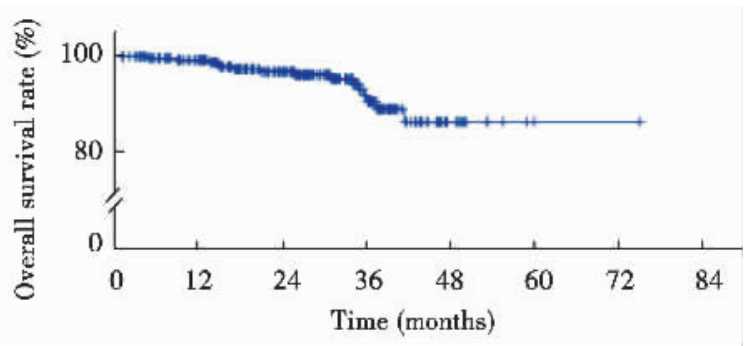

Figure 2 Overall survival curve of the 276 colorectal carcinoma patients

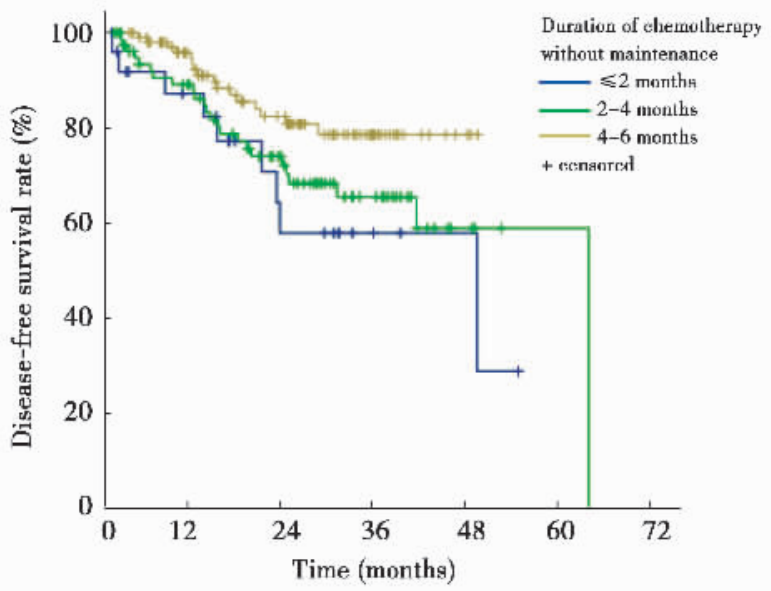

Figure 3 The effect of duration of adjuvant chemotherapy excluding xeloda or 5-FU/CF on 3-year disease-free survival of the 276 colorectal carcinoma patients 


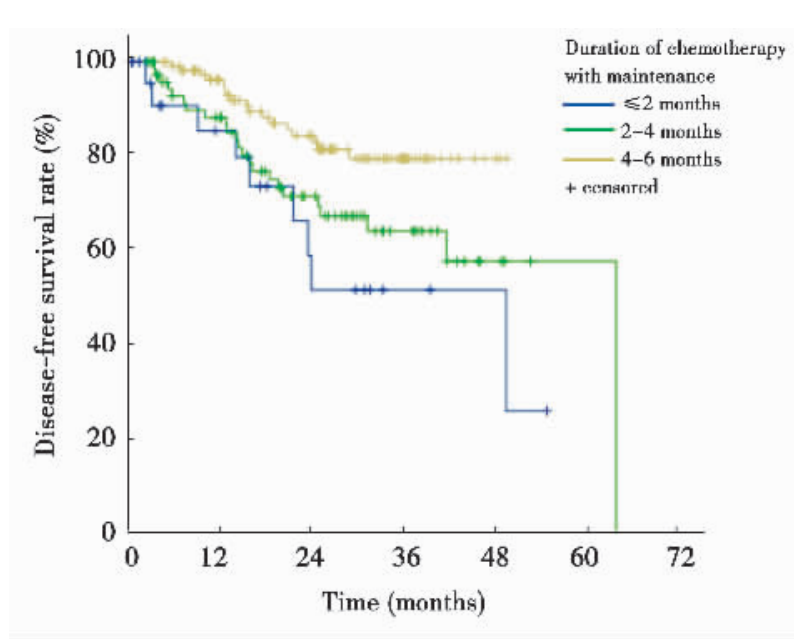

Figure 4 The effect of duration of adjuvant chemotherapy including xeloda or 5-FU/CF on 3-year disease-free survival of the 276 colorectal carcinoma patients

TNM stage $(\mathrm{P}<0.001), \quad \mathrm{N}$ stage $\quad(\mathrm{P}<0.001)$, pathologic differentiation $(\mathrm{P}=0.001)$ and duration of adjuvant chemotherapy (including the duration of xeloda or 5-FU/CF maintenance)
$(\mathrm{P}=0.01) \quad$ were all prognosis factors for the 3-year disease-free survival of colorectal carcinoma patients; while $\mathrm{T}$ stage, location of tumor, tumor diameter of $>5 \mathrm{~cm}$, CEA expression in tumor tissue, preoperative serum level of CEA, and the regimens of adjuvant chemotherapy (mFOLFOX6 or XELOX) had no impact on the 3-year disease-free survival $(\mathrm{P}>0$. 05).

Multivariate analysis showed that only age $(\mathrm{P}<0.001)$, gender $(\mathrm{P}=0.001), \mathrm{N}$ stage $(\mathrm{P}=$ 0.002), pathologic differentiation $(\mathrm{P}=0.043)$ and duration of adjuvant chemotherapy $(\mathrm{P}=$ 0.032) were independent prognosis factors of 3 -year disease-free survival (Table 2).

The 3-year disease-free survival rates were $71.0 \%$ for the 216 patients who received oxaliplatin-including regimens and $72.0 \%$ for the 60 patients who received oxaliplatin-free regimens $(\mathrm{P}=0.925)$.

Among the 216 patients who received oxaliplatin-including regimens as adjuvant chemotherapy, only 101 had record of peripheral

Table 2 Multivariate analysis for 3-year disease-free survival of the 216 colorectal cancer patients

\begin{tabular}{|c|c|c|c|c|c|c|}
\hline Variable & B & $\mathrm{SE}$ & Wald & df & Sig. & $\operatorname{Exp}(B)$ \\
\hline Age & -0.731 & 0.209 & 12.220 & 1 & 0.000 & 0.481 \\
\hline Sex & -1.750 & 0.498 & 9.444 & 1 & 0.002 & 0.216 \\
\hline Performance status & 0.522 & 0.591 & 0.780 & 1 & 0.377 & 1.686 \\
\hline $\mathrm{N}$ stage & 0.959 & 0.308 & 9.728 & 1 & 0.002 & 2.609 \\
\hline Pathologic subtype & -0.674 & 0.333 & 4.111 & 1 & 0.043 & 0.510 \\
\hline Duration of chemotherapy & -0.618 & 0.288 & 4.610 & 1 & 0.032 & 0.539 \\
\hline Tumor size & -0.375 & 0.443 & 0.718 & 1 & 0.397 & 0.687 \\
\hline CEA level & 0.253 & 0.377 & 0.452 & 1 & 0.502 & 1.289 \\
\hline $\mathrm{T}$ stage & 0.454 & 0.388 & 1.369 & 1 & 0.242 & 1.575 \\
\hline Site & -0.027 & 0.154 & 0.030 & 1 & 0.862 & 0.974 \\
\hline
\end{tabular}

neuropathy: 21 (20.8\%) had no neuropathy, 34 $(33.7 \%)$ had grade I neuropathy, 37 (36.6\%) had grade II neuropathy and 9 (8.9\%) had grade III neuropathy. The patients who developed neuropathy had received a median of 4 cycles of chemotherapy. Till October 2008, the end of follow-up, $36(35.6 \%)$ patients still suffered from grade I-II peripheral neuropathy, and 16 of them complained of worse numbness in the feet than in the hands while 20 had a similar numbness in the hands and feet.

\section{Discussion}

The role of postoperative adjuvant chemotherapy in resectable colorectal carcinoma patients has been elucidated sufficiently in recent years. In 2004, the results of MOSAIC trial established the firm status of 6-month FOLFOX as adjuvant chemotherapy in stage III colon cancer patients. ${ }^{6}$ Meanwhile, the QUASAR trial recommended adjuvant chemotherapy for 
high-risk stage II colon cancer patients. ${ }^{7}$ Since the information of adjuvant chemotherapy for rectal carcinoma is deficient, the information for colon cancer is mainly used as references.

Before 1998, the duration of adjuvant chemotherapy for colon cancer was 1 year. In 1998, the North Central Cancer Treatment Group (NCCTG) and the National Cancer Institute of Canada assessed the efficacy of 6 versus 12 months of postoperative adjuvant chemotherapy, and found that 12 months of chemotherapy did not have any benefits over 6 months of treatment. $^{8}$ The question of shortening the duration of adjuvant chemotherapy was further addressed in a randomized study conducted by Saini et al. ${ }^{9}$ In their trial, the patients with resectable Dukes' B and $\mathrm{C}$ colorectal cancer were randomly assigned to receive 5-FU and leucovorin (Mayo Clinic schedule) for 6 months or protracted venous infusion (PVI) of 5-FU for 12 weeks, the 12-week treatment resulted in higher recurrence-free survival rate and less toxicity as compared with 6-month treatment. Although the results are encouraging, the clinical application of 12 -week treatment is limited due to the small sample size (716 cases) of the trial. Six months of adjuvant chemotherapy is still the standard nowadays.

It was reported that there was a linear relationship between 3-year disease-free survival rate and 5-year overall survival rate in colon cancer patients, ${ }^{10}$ therefore, the 3-year disease-free survival rate can be used to predict 5 -year overall survival rate and it is good for the early observation of treatment effect. In our study, we used the 3-year disease-free survival rate for analysis.

In our study, the 216 patients who received oxaliplatin-including regimens were divided into 3 groups according to the duration of adjuvant chemotherapy. Both univariate and multivariate analyses showed that the duration of adjuvant chemotherapy was an independent factor of 3-year disease-free survival in colorectal carcinoma patients. The 3-year disease-free survival rate of the patients who received 4-6 months of adjuvant chemotherapy was $79.4 \%$, similar to the result of the MOSAIC trial. ${ }^{6}$

The records of neuropathy caused by oxaliplatin are less in our study than in the MOSAIC trial. ${ }^{6}$ Since it was a retrospective study and some of the patients could not remember the condition of neutropathy clearly. However, in both our study and the MOSAIC trial, the occurrence rate of grade III neuropathy was about $10 \%$ and it should not be the main reason preventing patients from receiving 6-month adjuvant chemotherapy.

In our study, only $22.7 \%$ of the patients finished 6-month adjuvant chemotherapy and the percentage was even smaller $(16.2 \%)$ when only the patients who received oxaliplatin-including regimen were counted. Over $60 \%$ of the patients followed the physicians decision to stop chemotherapy after 6 cycles of mFOLFOX6 or XELOX regimen, while only $6.5 \%$ of the patients stopped the chemotherapy due to intolerable toxicity. It is therefore necessary for the physicians to realize the role of adjuvant chemotherapy duration on the 3-year disease-free survival.

In our study, some of the patients could not tolerate 6-month treatment of oxaliplatin and used xeloda or 5-FU/CF as maintenance. Neglecting the duration of maintenance, the 3-year disease-free survival rates had no difference among the three groups of patients divided according to the duration of adjuvant chemotherapy. However, considering the duration of maintenance, the difference became significant. Since the sample size was small, we could not directly analyze the effect of maintenance chemotherapy on disease-free survival time for the patients who could not tolerate oxaliplatin. However, we could deduce that the maintenance of xeloda or 5-FU/CF may improve the 3-year disease-free survival rate of these patients.

Both univariate and multivariate analyses showed that age, gender, $\mathrm{N}$ stage and differentiation were independent prognostic factors of 3-year disease-free survival. Female patients had a longer disease-free survival than males. It was also reported that being male was a poor prognostic factor in rectal cancer patients 
who received preoperative neoadjuvant radiochemotherapy. ${ }^{11}$ However, the underlying mechanism is still unclear. Whether estrogen had the protective function in colorectal carcinoma patients or not should be further investigated. Whether being male is a high risk factor for stage II colorectal patients to receive adjuvant chemotherapy also needs to be further investigated. Moreover, the 3-year disease-free survival rates were similar in patients receiving oxaliplatin-including regimen and non-oxaliplatin regimen. The percentage of stage II patients was higher in non-oxaliplatin group than in oxaliplatin-including group $(88.3 \%$ vs $33.4 \%$ ). Though only 60 cases in non-oxaliplatin group were available for analysis, we can still recommend stage II colorectal carcinoma patients to receive 5-FU/CF or xeloda as adjuvant chemotherapy.

In conclusion, the duration of adjuvant chemotherapy is an independent prognostic factor for 3-year disease-free survival of colorectal carcinoma patients. Patients who received 4-6 months of adjuvant chemotherapy had a longer 3-year disease-free survival than those only received $\leq 4$ months of adjuvant chemotherapy.

\section{References}

[1] Cao KJ, Fan QY, Liu YL, et al. Cancer incidence and mortality in Guangzhou City from 2000 to 2002 [J]. Ai Zheng, 2008,27(3):225-230. [in Chinese]

[2] Gill S, Loprinzi CL, Sargent DJ, et al. Pooled analysis of fluorouracil-based adjuvant therapy for stage II and III colon cancer: who benefits and by how much? [J]. J Clin Oncol, 2004, 22(10) : 1797-1806.
[3] Benson AB 3rd, Schrag D, Somerfield MR, et al. American Society of Clinical Oncology recommendations on adjuvant chemotherapy for stage II colon cancer [J]. J Clin Oncol, 2004, 22 (16) :3408-3419.

[4] Compton CC, Fielding LP, Burgart LJ, et al. Prognostic factors in colorectal cancer. College of American Pathologists Consensus Statement 1999 [J]. Arch Pathol Lab Med, 2000, 124 (7) : 979-994.

[5] Extra JM, Espie M, Calvo F, et al. Phase I study of oxaliplatin in patients with advanced cancer $[\mathrm{J}]$. Cancer Chemother Pharmacol, 1990,25 (4) :299-303.

[6] Andre T, Boni C, Mouredji-Bardint L, et al. Oxaliplatin, fluorouracil, and leucovorin as adjuvant treatment for colon cancer [J]. N Engl J Med, 2004,350(23): 2343.

[7] QUASAR Collaborative Group, Gray R, Barnwell J, McConkey C, et al. Adjuvant chemotherapy versus observation in patients with colorectal cancer: a randomized study $[\mathrm{J}]$. Lancet, 2007, 370 (9604) : 2020-2029.

[8] O'Connell MJ, Laurie JA, Kahn M, et al. Prospectively randomized trial of postoperative adjuvant chemotherapy in patients with high-risk colon cancer [J]. J Clin Oncol, 1998, 16(1) : 295-300.

[9] Saini A, Norman AR, Cunningham D, et al. Twelve weeks of protracted venous infusion of fluorouracil (5-FU) is as effective as 6 months of bolus 5-FU and folinic acid as adjuvant treatment in colorectal cancer $[\mathrm{J}]$. Br J Cancer, $2003,88(12): 1859-1865$.

[10] Sargent D, Wieand S, Goldberg R, et al. 3 Year DFS vs. 5 year $\mathrm{OS}$ as an endpoint for adjuvant colon cancer studies: data from randomized trials. Rockville, Md.: Food and Drug Administration, November 2003. (Accessed May 10, 2004, at. http://www.fda.gov/cder/drug/cancer_endpoints/Sargent/ index.htm.).

[11] Díaz-González JA, Calvo FA, Cortés J, et al. Prognostic factors for disease-free survival in patients with T3-4 or $\mathrm{N}+$ rectal cancer treated with preoperative chemoradiation therapy, surgery, and intraoperative irradiation $[\mathrm{J}]$. Int J Radiat Oncol Biol Phys, 2006,64(4):1122-1128. 